\title{
Evaluation subgroups of a map and the rationalized $G$-sequence
}

\author{
O. Maphane
}

\begin{abstract}
In this paper, we determine, in terms of the Sullivan models, rational evaluation subgroups of the inclusion $\mathbb{C} P(n) \hookrightarrow$ $\mathbb{C} P(n+k)$ between complex projective spaces and, more generally, the $G$-sequence of the homotopy monomorphism $\iota: X \hookrightarrow Y$ between simply connected formal homogeneous spaces for which $\pi_{*}(Y) \otimes \mathbb{Q}$ is finite dimensional.
\end{abstract}

Key Words: Evaluation subgroups, Gottlieb group, $G$-sequence Mathematics Subject Classification 2010: 55P62, 54C35

\section{Introduction}

Let us remind the notion of a Gottlieb group (see [3]). Given a based CWcomplex $X$, an element $\alpha \in \pi_{n}(X)$ is a Gottlieb element of $X$ if $\left(\alpha, i d_{X}\right)$ : $X \vee S^{n} \rightarrow X$ extends to $\tilde{\alpha}: X \times S^{n} \rightarrow X$. The set $G_{n}(X)$ of all Gottlieb elements $\alpha \in \pi_{n}(X)$ is called the $n$-th Gottlieb group of $X$ or the $n$-th evaluation subgroup of $\pi_{n}(X)$.

Gottlieb groups play a profound role in topology, covering spaces, fixed point theory, homotopy theory of fibrations, and other fields. For instance, the triviality of Gottlieb groups is related to the cross section problem of fibrations.

Further, let $f: X \rightarrow Y$ be a based map of simply connected finite CWcomplexes. As it was shown in [4], the evaluation at the basepoint of $X$ gives the evaluation map $\omega: \operatorname{Map}(X, Y ; f) \rightarrow Y$, where $\operatorname{Map}(X, Y ; f)$ is the component of $f$ in the space of mappings from $X$ to $Y$. The image of the homomorphism induced in homotopy groups

$$
\omega_{\sharp}: \pi_{*} \operatorname{Map}(X, Y ; f) \rightarrow \pi_{*}(Y)
$$


is called the $n$-th evaluation subgroup of $p$ and it is denoted by $G_{n}(Y, X ; p)$. Note that if $f=i d_{X}$, the $\operatorname{space} \operatorname{Map}(X, Y ; f)$ is the monoid $\operatorname{aut}_{1}(X)$ of selfequivalences of $X$ homotopic to the identity of $X$, then ev $: \operatorname{aut}_{1}(X) \rightarrow X$ is the evaluation map, and the image of the induced homomorphism

$$
e v_{\sharp}: \pi_{*}\left(\operatorname{aut}_{1}(X)\right) \rightarrow \pi_{*}(X)
$$

is $G_{n}(X)$, i.e., the $n$-th Gottlieb group.

In [10, Woo and Lee studied the relative evaluation subgroups $G_{n}^{r e l}(X, Y ; p)$ and proved that they fit in a sequence

$$
\cdots \rightarrow G_{n+1}^{r e l}(X, Y ; f) \rightarrow G_{n}(X) \rightarrow G_{n}(X, Y ; f) \rightarrow \cdots
$$

called the $G$-sequence of $f$. This sequence is exact in some cases, for instance, if $f$ is a homotopy monomorphism.

Recently, Smith and Lupton [4] identified the homomorphism induced on rational homotopy groups by the evaluation map $\omega: \operatorname{Map}(X, Y ; f) \rightarrow Y$ in terms of a map of complexes of derivations constructed directly from the Sullivan minimal model of $f$. In [5, 6], rationalized evaluation subgroups of mapping spaces between Complex Grassmann manifolds $G_{k, n}(\mathbb{C})$, which are a generalization of complex projective spaces, were studied.

In this paper, we use a map of complexes of derivations of minimal Sullivan models of mapping spaces to compute rational relative Gottlieb groups of the inclusion $\mathbb{C} P(n) \hookrightarrow \mathbb{C} P(n+k)$. More generally, we consider the inclusion $\iota: X \hookrightarrow Y$ between simply connected formal homogeneous spaces for which $\pi_{*}(Y) \otimes \mathbb{Q}$ is finite dimensional.

\section{Preliminaries}

Through this paper, we rely on the theory of minimal Sullivan models in rational homotopy theory for which [1] is our standard reference. All vector spaces and algebras are taken over a field $\mathbb{Q}$ of rational numbers. We start with recalling some definitions.

Definition 1.1 A commutative graded differential algebra (cdga) is a graded algebra $(A, d)$ such that $x y=(-1)^{|x||y|} y x$ and $d(x y)=(d x) y+(-1)^{|p q|} x(d y)$ for all $x \in A^{p}, y \in A^{q}$. It is said to be connected if $H^{0}(A) \cong \mathbb{Q}$. If $V=\oplus_{i \geq 1} V^{i}$ with $V^{\text {even }}:=\oplus_{i \geq 1} V^{2 i}$ and $V^{\text {odd }}:=\oplus_{i \geq 1} V^{2 i-1}$, then $\wedge V$ denotes the free commutative graded algebra defined by the tensor product

$$
\wedge V=S\left(V^{\text {even }}\right) \otimes E\left(V^{\text {odd }}\right),
$$

where $S\left(V^{\text {even }}\right)$ is the symmetric algebra on $V^{\text {even }}$ and $E\left(V^{\text {odd }}\right)$ is the exterior algebra on $V^{\text {odd }}$. 
Definition 1.2 A Sullivan algebra is a commutative differential graded algebra $(\wedge V, d)$ where $V=\cup_{k \geq 0} V(k)$ and $V(0) \subset V(1) \cdots$ such that $d V(0)=0$ and $d V(k) \subset \wedge V(k-1)$. It is called minimal if $d V \subset \wedge^{\geq 2} V$.

If $(A, d)$ is a cdga of which the cohomology is connected and finite dimensional in each degree, then there always exists a quasi-isomorphism from a Sullivan algebra $(\wedge V, d)$ to $(A, d)$ [1]. To each simply connected space, Sullivan associates a cdga $A_{P L}(X)$ of rational polynomial differential forms on $X$ that uniquely determines the rational homotopy type of $X$ [8]. A minimal Sullivan model of $X$ is a minimal Sullivan model of $A_{P L}(X)$. More precisely, $H^{*}(\wedge V, d) \cong H^{*}(X ; \mathbb{Q})$ as graded algebras and $V \cong \pi_{*}(X) \otimes \mathbb{Q}$ as graded vector spaces.

Let $(A, d)$ be a cdga. A derivation $\theta$ of degree $k$ is a linear mapping $\theta: A^{n} \rightarrow A^{n-k}$ such that $\theta(a b)=\theta(a) b+(-1)^{k|a|} a \theta(b)$. Denote by $\operatorname{Der}_{k} A$ the vector space of all derivations of degree $k$ and $\operatorname{Der} A=\oplus_{k} \operatorname{Der}_{k} A$. The commutator bracket induces a graded Lie algebra structure on $\operatorname{Der} A$. Moreover, (Der $A, \delta)$ is a differential graded Lie algebra (see, for example, [8]), with the differential $\delta$ defined in the usual way by

$$
\delta \theta=d \circ \theta+(-1)^{k+1} \theta \circ d .
$$

Let $(\wedge V, d)$ be a Sullivan algebra where $V$ is spanned by $\left\{v_{1} \ldots, v_{k}\right\}$. Then, Der $\wedge V$ is spanned by $\theta_{1}, \ldots, \theta_{k}$, where $\theta_{i}$ is the unique derivation of $\wedge V$ defined by $\theta_{i}\left(v_{j}\right)=\delta_{i j}$. The derivation $\theta_{i}$ will be denoted by $\left(v_{i}, 1\right)$. It is known (see [1]), that an element $v \in V \cong \pi_{*}(X) \otimes \mathbb{Q}$ is a Gottlieb element of $\pi_{*}(X) \otimes \mathbb{Q}$ if and only if there is a derivation $\theta$ of $\wedge V$ satisfying $\theta(v)=1$ and such that $\delta \theta=0$. Let $\phi:(A, d) \rightarrow(B, d)$ be a morphism of cdga's. A $\phi$-derivation of degree $k$ is a linear mapping $\theta: A^{n} \rightarrow B^{n-k}$ for which $\theta(a b)=\theta(a) \phi(b)+(-1)^{k|a|} \phi(a) \theta(b)$. We consider only derivations of positive degree. Denote by $\operatorname{Der}_{n}(A, B ; \phi)$ the vector space of $\phi$-derivations of degree $n$ for $n>0$, and by $\operatorname{Der}(A, B ; \phi)=\oplus_{n} \operatorname{Der}_{n}(A, B ; \phi)$ the graded vector space of all $\phi$-derivations.

The differential graded vector space of $\phi$-derivations is denoted by $(\operatorname{Der}(A, B ; \phi), \partial)$, where the differential $\partial$ is defined by

$$
\partial \theta=d_{B} \circ \theta+(-1)^{k+1} \theta \circ d_{A} .
$$

In the case $A=B$ and $\phi=1_{B}$, the vector space $(\operatorname{Der}(B, B ; 1), \partial)$ is just a usual differential graded Lie algebra of derivations on the cdga $B$ (see [4]). Note that, there is an isomorphism of graded vector spaces

$$
\operatorname{Der}(A, B ; \phi) \cong \operatorname{Hom}(V, B) \text {. }
$$

If $\left\{v_{i}\right\}$ is a basis of $V$, then the vector space $\operatorname{Der}(A, B ; \phi)$ is spanned by the unique $\phi$-derivation $\theta$ denoted by $\left(v_{i}, b_{i}\right)$ and $\left(v_{i}, 1\right)$ such that

$$
\left\{\begin{array}{l}
\theta_{i}\left(v_{i}\right)=b_{i}, \\
\theta_{i}\left(v_{j}\right)=0, \quad i \neq j, b_{i} \in B .
\end{array}\right.
$$


It was shown in [4], that a pre-composition with $\phi$ gives a chain complex map $\phi^{*}: \operatorname{Der}(B, B ; 1) \rightarrow \operatorname{Der}(A, B ; \phi)$, and a post-composition with the augmentation $\varepsilon: B \rightarrow \mathbb{Q}$ gives a chain complex map $\varepsilon_{*}: \operatorname{Der}(A, B ; \phi) \rightarrow$ $\operatorname{Der}(A, \mathbb{Q} ; \varepsilon)$. The evaluation subgroup of $\phi$ is defined as follows

$$
G_{n}(A, B ; \phi)=\operatorname{Im}\left\{H\left(\varepsilon_{*}\right): H_{n}(\operatorname{Der}(A, B ; \phi)) \rightarrow H_{n}(\operatorname{Der}(A, \mathbb{Q} ; \varepsilon))\right\} .
$$

In the case $A=B$ and $\phi=1_{B}$, we get the Gottlieb group of $(B, d)$ defined as

$$
G_{n}(B)=\operatorname{Im}\left\{H\left(\varepsilon_{*}\right): H_{n}(\operatorname{Der}(B, B ; 1)) \rightarrow H_{n}(\operatorname{Der}(B, \mathbb{Q} ; \varepsilon))\right\} .
$$

In particular, $G_{n}(B) \cong G_{n}\left(X_{\mathbb{Q}}\right)$ if $B$ is the minimal Sullivan model of a simply connected space $X$ [1, Proposition 29.8].

Definition 1.3 A simply connected space $X$ is called formal (see [2]) if there is a quasi-isomorphism $(\wedge V, d) \rightarrow H^{*}(\wedge V, d)$, where $(\wedge V, d)$ is the minimal Sullivan model of $X$.

Examples of formal spaces include spheres, projective complex spaces, homogeneous spaces $G / H$ where $G$ and $H$ have same rank, and compact Kähler manifolds.

\section{Evaluation subgroups of a map and the $G$ - sequence}

Consider the inclusion $\mathbb{C} P(n) \hookrightarrow \mathbb{C} P(n+k)$. In [2], the minimal model of $\mathbb{C} P(n)$ is given by $\left(\wedge\left(x_{2}, x_{2 n+1}\right), d\right)$ where $d x_{2}=0, d x_{2 n+1}=x_{2}^{n+1}$, and the minimal model of $\mathbb{C} P(n+k)$ is given by $\left.\left(\wedge y_{2}, y_{2(n+k)+1}\right), d\right)$ with $d y_{2}=$ $0, d y_{2(n+k)+1}=y_{2}^{n+k+1}$. Moreover, the inclusion $\mathbb{C} P(n) \hookrightarrow \mathbb{C} P(n+k)$ has a model of the form

$$
\phi: A=\wedge y_{2} /\left(y_{2}^{n+k+1}\right) \rightarrow \wedge x_{2} /\left(x_{2}^{n+1}\right)=B
$$

where $\phi\left(y_{2}\right)=x_{2}$.

Let $X$ be a homogeneous space of which $(\wedge V, d)=\left(\wedge\left(V_{0} \oplus V_{1}\right), d\right)$ is its minimal Sullivan model, where $V$ is finite dimensional and $d V_{0}=0, d V_{1} \subseteq$ $\wedge V_{0}$. Write $V_{0}^{\text {even }}=\mathbb{Q}<p_{1}, \ldots, p_{q}>=P, V_{0}^{\text {odd }}=\mathbb{Q}<w_{1}, \ldots, w_{r}>=W$ and $V_{1}^{\text {odd }}=\mathbb{Q}<y_{1}, \ldots, y_{k}>=Y$, so that $\left(\wedge\left(V_{0} \oplus V_{1}\right), d\right) \stackrel{\cong}{\rightarrow}(\wedge(P \oplus Y), d) \otimes$ $(\wedge W, 0)$ and $d P=0, d Y \subseteq \wedge P$. The associated minimal Sullivan model $(\wedge V, d)$ is called a pure Sullivan algebra. Homogeneous spaces are pure. Further, since $X$ is a formal homogeneous space, we have

$$
H^{*}(\wedge V, d)=\frac{\wedge\left(p_{1}, \ldots, p_{q}\right)}{\left(\alpha_{1}, \ldots, \alpha_{k}\right)} \otimes \wedge\left(w_{i}, \ldots, w_{r}\right),
$$


where $\left(\alpha_{1}, \ldots, \alpha_{k}\right)$ is a regular sequence in $\wedge P$. Hence, $X$ as a formal homogeneous space admitting a minimal Sullivan model of the form $(\wedge V, d)=$ $(\wedge(P \oplus Y), d) \otimes(\wedge W, 0)$, where $d P=0, d y_{k}=\alpha_{k}$.

We study the evaluation subgroups of $\phi$ and, more generally, the inclusion $\iota$ between formal spaces. We have the following results.

Theorem 2.1 Let $B=\left(\wedge\left(x_{2}, x_{2 n+1}\right), d\right)$. Then $G_{n}(B)=\left\langle\left[x_{2 n+1}^{*}\right]\right\rangle$.

Proof. Note that $\operatorname{Der}(B, B ; 1)=\oplus_{i=0}^{n} \mathbb{Q} \alpha_{2 i+1} \oplus \mathbb{Q} \alpha_{2}$, where $\alpha_{2}$ is the derivation taking $x_{2}$ to one and $\alpha_{2 i+1}$ is the derivation taking $x_{2 n+1}$ to $x_{2}^{n-i}$ for $i=1, \ldots, n$. The differential is given by $\delta \alpha_{2 i+1}=0$ and $\delta \alpha_{2}=(n+1) \alpha_{1}$. Hence, for $1 \leq i \leq n,\left[\alpha_{2 i+1}\right]$ represents a non-zero homology class in $H_{*}(\operatorname{Der}(B, B ; 1))$. Moreover, $\varepsilon_{*}\left(\alpha_{2 i+1}\right)=x_{2 i+1}^{*}$. As $\mathbb{C} P(n)$ is a finite CWcomplex, then $G_{\text {even }}(B)=0$ (see [1, Page 379]). Hence, $G_{n}(B)=\left\langle\left[x_{2 i+1}^{*}\right]\right\rangle$.

Theorem 2.2 Consider the inclusion $\mathbb{C} P(n) \hookrightarrow \mathbb{C} P(n+k)$, and let $\phi$ : $A \rightarrow B$ be its Sullivan model. Then $G_{*}(A, B ; \phi)=\left\langle\left[y_{2(n+k)+1}^{*}\right]\right\rangle$.

Proof. Define $\theta_{2(n+k)+1}=\left(y_{2(n+k)+1}, 1\right)$ in $\operatorname{Der}(A, B ; \phi)$ and the differential given by $\partial \theta_{2(n+k)+1}=0$. Note that $\left[\theta_{2(n+k)+1}\right]$ is a non-zero homology class in $H_{*}(\operatorname{Der}(A, B ; \phi))$. A straightforward calculation shows that $\theta_{2}=\left(x_{2}, 1\right)$ is not a cycle in $\operatorname{Der}(A, B ; \phi)$. Moreover, $H\left(\varepsilon_{*}\right)\left(\left[\theta_{2(n+k)+1}\right]\right)=\left[y_{2(n+k)+1}^{*}\right] \in$ $G_{2(n+k)+1}(A, B ; \phi)$. It then follows that $G_{*}(A, B ; \phi)=\left\langle\left[y_{2(n+k)+1}^{*}\right]\right\rangle$.

Definition 2.3 Let $\phi: A \rightarrow B$ be a map of differential graded vector spaces. $A$ differential graded vector space, $\operatorname{Rel}_{*}(\phi)$, called the mapping cone of $\phi$ (see, for example, [9, 4]) is defined by $\operatorname{Rel}_{n}(\phi)=A_{n-1} \oplus B_{n}$ for all $n>1$, with the differential $\delta(a, b)=\left(-d_{A}(a), \phi(a)+d_{B}(b)\right)$. There are inclusion and projection chain maps $J: B_{n} \rightarrow \operatorname{Rel}_{n}(\phi)$ and $P: \operatorname{Rel}_{n}(\phi) \rightarrow A_{n-1}$ defined by $J(w)=(0, w)$ and $P(a, b)=a$, respectively. These yields a short exact sequence of chain complexes

$$
0 \rightarrow B_{*} \stackrel{J}{\rightarrow} \operatorname{Rel}_{*}(\phi) \stackrel{P}{\rightarrow} A_{*-1} \rightarrow 0
$$

and a long exact homology sequence of $\phi$

$$
\cdots \rightarrow H_{n+1}(\operatorname{Rel}(\phi)) \stackrel{H(P)}{\rightarrow} H_{n}(A) \stackrel{H(\phi)}{\rightarrow} H_{n}(B) \stackrel{H(J)}{\rightarrow} H_{n}(\operatorname{Rel}(\phi)) \rightarrow \cdots
$$

whose connecting homomorphism is $H(\phi)$.

Following [4], we consider a commutative diagram of differential graded vector spaces

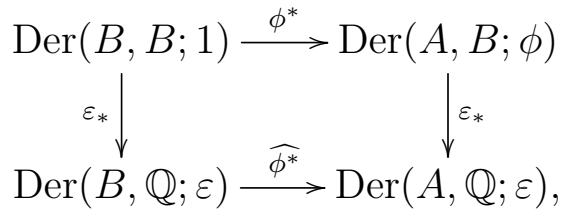


where $\varepsilon$ is the augmentation of either $A$ or $B$. On passing to homology and using the naturality of the mapping cone construction, we obtain the following homology ladder for $n \geq 2$,

$$
\begin{aligned}
\cdots & \rightarrow H_{n+1}\left(\operatorname{Rel}\left(\phi^{*}\right)\right) \stackrel{H(P)}{\longrightarrow} H_{n}(\operatorname{Der}(B, B ; 1)) \stackrel{H\left(\phi^{*}\right)}{\longrightarrow} H_{n}(\operatorname{Der}(A, B ; \phi)) \rightarrow \cdots \\
& H\left(\varepsilon_{*}, \varepsilon_{*}\right) \downarrow \\
\cdots & \rightarrow H_{n+1}\left(\operatorname{Rel}\left(\widehat{\phi}^{*}\right)\right) \stackrel{H(\widehat{P})}{\longrightarrow} H_{n}(\operatorname{Der}(B, \mathbb{Q} ; \varepsilon)) \stackrel{H\left(\varepsilon_{*}\right) \mid}{\stackrel{H\left(\widehat{\phi}^{*}\right.}{\longrightarrow}} H_{n}(\operatorname{Der}(A, \mathbb{Q} ; \varepsilon)) \rightarrow \cdots
\end{aligned}
$$

The $n$-th relative evaluation subgroup of $\phi$ is defined as follows

$$
G_{n}^{r e l}=\operatorname{Im}\left\{H\left(\varepsilon_{*}, \varepsilon_{*}\right): H_{n}\left(\operatorname{Rel}\left(\phi^{*}\right)\right) \rightarrow H_{n}\left(\operatorname{Rel}\left(\widehat{\phi}^{*}\right)\right)\right\} .
$$

The $G$-sequence of the map $\phi: A \rightarrow B$ is given by the sequence

$$
\cdots \stackrel{H(\widehat{J})}{\rightarrow} G_{n+1}^{r e l}(A, B ; \phi) \stackrel{H(\widehat{P})}{\rightarrow} G_{n}(B) \stackrel{H\left(\widehat{\phi^{*}}\right)}{\rightarrow} G_{n}(A, B ; \phi) \stackrel{H(\widehat{J})}{\rightarrow} \cdots
$$

which ends in $G_{2}(A, B ; \phi)$. Moreover, as it was shown in [4, Theorem 3.5], this can be applied to the Sullivan model $\phi: A \rightarrow B$ of the map $f: X \rightarrow Y$.

Theorem 2.4 Consider the inclusion $\mathbb{C} P(n) \hookrightarrow \mathbb{C} P(n+k)$, and let $\phi: A \rightarrow$ $B$ be its Sullivan model. Then $G_{*}^{r e l}(A, B ; \phi)=\left\langle\left[\left(x_{2 n+1}^{*}, 0\right)\right],\left[\left(0, y_{2(n+k)+1}^{*}\right)\right]\right\rangle$.

Proof. We will use the following diagram presented in [4]:

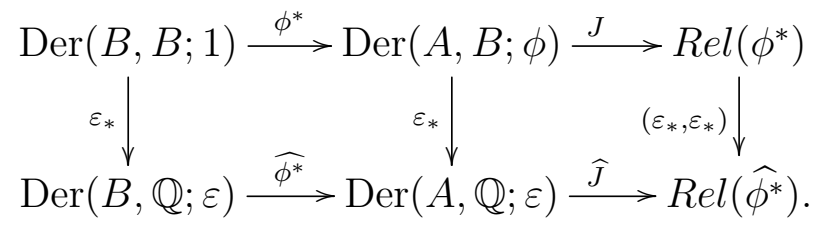

Consider $\alpha_{2 n+1}=\left(x_{2 n+1}, 1\right)$ in $\operatorname{Der}(B, B ; 1)$ and $\theta_{2(n+k)+1}=\left(y_{2(n+k)+1}, 1\right)$ in $\operatorname{Der}(A, B ; \phi)$. Then

$$
\phi^{*}\left(\alpha_{2 n+1}\right)= \begin{cases}\theta_{2(n+k)+1} & \text { if } 2 n+1=2(n+k)+1, \\ 0 & \text { if } 2 n+1 \neq 2(n+k)+1,\end{cases}
$$

such that

$$
D\left(\alpha_{2 n+1}, 0\right)= \begin{cases}\left(0, \theta_{2(n+k)+1}\right) & \text { if } 2 n+1=2(n+k)+1, \\ (0,0) & \text { if } 2 n+1 \neq 2(n+k)+1,\end{cases}
$$

and $D\left(0, \theta_{2(n+k)+1}\right)=(0,0)$. Thus, if $2 n+1 \neq 2(n+k)+1$, then $\left[\left(\alpha_{2 n+1}, 0\right)\right]$ and $\left[\left(0, \theta_{2(n+k)+1}\right)\right]$ are non-zero homology classes in $H_{*}\left(\operatorname{Rel}\left(\phi^{*}\right)\right)$. Moreover, $H\left(\varepsilon_{*}, \varepsilon_{*}\right)\left(\left[\left(\alpha_{2 n+1}, 0\right)\right]\right)=\left[\left(x_{2 n+1}^{*}, 0\right)\right]$ for $2 n+1 \neq 2(n+k)+1$, and in the same way, $H\left(\varepsilon_{*}, \varepsilon_{*}\right)\left(\left[\left(0, \theta_{2(n+k)+1}\right)\right]\right)=\left[\left(0, y_{2(n+k)+1}^{*}\right)\right]$. A straightforward calculations shows that for $2 n+1 \neq 2(n+k)+1,\left[\left(x_{2 n+1}^{*}, 0\right)\right]$ and $\left[\left(0, y_{2(n+k)+1}^{*}\right)\right]$ $\operatorname{span} H\left(\varepsilon_{*}, \varepsilon_{*}\right)$. 
From the theorem above, it follows that the $G$-sequence reduces to

$$
\begin{gathered}
0 \rightarrow G_{2(n+k)+1}(A, B ; \phi) \stackrel{H(J)}{\longrightarrow} G_{2(n+k)+1}^{r e l}(A, B ; \phi) \rightarrow 0, \\
0 \rightarrow G_{2 n+1}(B) \stackrel{H\left(\phi^{*}\right)}{\longrightarrow} G_{2 n+1=2(n+k)+1}(A, B ; \phi) \rightarrow 0, \\
\quad 0 \rightarrow G_{2 n+1}^{r e l}(A, B ; \phi) \stackrel{H(P)}{\longrightarrow} G_{2 n+1}(B) \rightarrow 0,
\end{gathered}
$$

and it is exact.

Example 1 Consider the inclusion $\mathbb{C} P(1) \hookrightarrow \mathbb{C} P(2)$. A Sullivan model of the inclusion is given by

$$
\left.\phi: A=\left(\wedge\left(y_{2}, y_{5}\right), d\right) \rightarrow\left(\wedge x_{2}, x_{3}\right), d\right)=B
$$

where $d y_{2}=0, d y_{3}=y_{2}^{2}, d x_{2}=0$ and $d x_{3}=x_{2}^{2}$. Moreover, $\phi\left(y_{2}\right)=x_{2}$ and $\phi\left(y_{5}\right)=x_{2} x_{3}$.

Determine $G_{*}^{\text {rel }}(A, B ; \phi)$ as follows. Consider $\alpha_{3}=\left(x_{3}, 1\right) \in \operatorname{Der}(B, B ; 1)$ and $\theta_{5}=\left(y_{5}, 1\right) \in \operatorname{Der}(A, B ; \phi)$. Then $\phi^{*}\left(\alpha_{3}\right)=0$. Thus, $D\left(\alpha_{3}, 0\right)=(0,0)$ and $D\left(0, \theta_{3}\right)=(0,0)$. Hence, $\left[\left(\alpha_{3}, 0\right)\right]$ and $\left[\left(0, \theta_{5}\right]\right.$ are non-zero homology classes. Further, $\left(\varepsilon_{*}, \varepsilon_{*}\right)\left(\alpha_{3}, 0\right)=\left(x_{3}^{*}, 0\right)$ and $\left(\varepsilon_{*}, \varepsilon_{*}\right)\left(0, \theta_{5}\right)=\left(0, y_{5}^{*}\right)$. Therefore,

$$
G_{n}^{r e l}(A, B ; \phi)=\left\langle\left[\left(0, y_{3}^{*}\right)\right],\left[\left(x_{3}^{*}, 0\right)\right]\right\rangle
$$

Proposition 2.5 Let $(\wedge V, d)=\left(\wedge\left(V_{0} \oplus V_{1}\right), d\right)$ be a minimal Sullivan model, where $V$ is finite dimensional with $d V_{0}=0, d V_{1} \subseteq \wedge V_{0}, V_{0}^{\text {even }}=$ $\mathbb{Q}<p_{1}, \ldots, p_{q}>, V_{0}^{\text {odd }}=\mathbb{Q}<w_{1}, \ldots, w_{r}>$, and $V_{1}^{\text {odd }}=\mathbb{Q}<y_{1}, \ldots, y_{k}>$. Then the generators $y_{1}, \ldots, y_{k}$ and $w_{1}, \ldots, w_{r}$ are Gottlieb elements where the subscripts indicate degrees.

Proof. For $t \in\{1, \ldots, k\}$, let $\theta_{t}$ denotes the derivation of $\wedge V$, that is,

$$
\theta_{t}\left(y_{j}\right)= \begin{cases}0, & t \neq j \\ 1, & t=j\end{cases}
$$

It is easy to see that $\delta \theta_{t}\left(y_{t}\right)=0$. Hence, the generators $y_{t}, t \in\{1, \ldots, k\}$ are Gottlieb elements. It follows in the same way that the generators $w_{1}, \ldots, w_{r}$ are Gottlieb elements.

Theorem 2.6 Let $X$ be a simply connected formal homogeneous spaces for which $\pi_{*}(X) \otimes \mathbb{Q}$ is finite dimensional, and let $B=\left(\wedge\left(V_{0} \oplus V_{1}\right), d\right)$ be its minimal Sullivan model. Then $G_{*}(B)$ is generated by $\left\langle\left[y_{1}^{*}\right], \ldots,\left[y_{k}^{*}\right]\right\rangle$ and $\left\langle\left[w_{1}^{*}\right], \ldots,\left[w_{r}^{*}\right]\right\rangle$ as a vector space, where the subscripts indicate degrees. 
Proof. Consider a minimal Sullivan model $B=(\wedge V, d)=\left(\wedge\left(V_{0} \oplus V_{1}\right), d\right)$ of $X$ for which $V$ is finite dimensional. If $V_{1}=V_{1}^{\text {odd }}=\mathbb{Q}<y_{1}, \ldots, y_{k}>$, denote by $\left(y_{t}, 1\right)$ the derivation $\theta_{t}$ such that $\theta_{t}\left(y_{j}\right)=1, t=j$, and zero otherwise. Then $\delta \theta_{t}\left(y_{t}\right)=0$. If $V_{0}=V_{0}^{\text {odd }}=\mathbb{Q}<w_{1}, \ldots, w_{r}>$, for $s \in\{1, \ldots, r\}$, define the derivation $\alpha_{s}=\left(w_{s}, 1\right)$ in the same way such that $\delta \alpha_{s}\left(w_{s}\right)=0$. Then, by Proposition 2.5, the generators $y_{1}, \ldots, y_{k}$ and $w_{1}, \ldots, w_{r}$ are Gottlieb elements. It follows from the definition of the Gottlieb group of $B$ that $\left[\theta_{1}\right], \ldots,\left[\theta_{k}\right]$ and $\left[\alpha_{1}\right], \ldots,\left[\alpha_{r}\right]$ are non-zero homology classes in $H_{*}(\operatorname{Der}(B, B ; 1))$. Also, $\varepsilon_{*}\left(\theta_{1}\right)=y_{1}^{*}, \ldots, \varepsilon_{*}\left(\theta_{k}\right)=y_{k}^{*}$ and $\varepsilon_{*}\left(\alpha_{1}\right)=w_{1}^{*}, \ldots, \varepsilon_{*}\left(\alpha_{r}\right)=w_{r}^{*}$. As $X$ is a simply connected finite CWcomplex, then $G_{\text {even }}(B)=0$ (see [1, Proposition 28.8]). Thus, $G_{*}(B)$ is generated by $\left\langle\left[y_{1}^{*}\right], \ldots,\left[y_{k}^{*}\right]\right\rangle$ and $\left\langle\left[w_{1}^{*}\right], \ldots,\left[w_{r}^{*}\right]\right\rangle$ as a vector space.

Theorem 2.7 Consider the homotopy monomorphism $\iota: X \hookrightarrow Y$ between simply connected formal homogeneous spaces for which $\pi_{*}(Y) \otimes \mathbb{Q}$ is finite dimensional, and let $\phi: \wedge V=\left(\wedge\left(V_{0} \oplus V_{1}\right), d\right) \rightarrow(B, d)$ be its Sullivan model. Then $G_{*}(\wedge V, B ; \phi)$ is generated as a vector space by $\left\langle\left[y_{p}^{*}\right], \ldots,\left[y_{k}^{*}\right]\right\rangle$ and $\left\langle\left[w_{q}^{*}\right], \ldots,\left[w_{r}^{*}\right]\right\rangle$ for some $p \in\{1, \ldots, k\}$ and $q \in\{1, \ldots, r\}$, which are determined by the homotopy monomorphism. Here, the subscripts indicate degrees.

Proof. Let $\phi: \wedge V=\left(\wedge\left(V_{0} \oplus V_{1}\right), d\right) \rightarrow(\wedge(B, d)$ be a Sullivan model for the homotopy monomorphism $\iota: X \hookrightarrow Y$, where $V$ is finite dimensional. Suppose that $V_{1}=V_{1}^{\text {odd }}=\mathbb{Q}<y_{1}, \ldots, y_{k}>$ and $V_{0}=V_{0}^{\text {odd }}=\mathbb{Q}<w_{1}, \ldots, w_{r}>$. Denote by $\left(y_{p}, 1\right)$ for $p \in\{1, \ldots, k\}$ and $\left(w_{q}, 1\right)$ for $q \in\{1, \ldots, r\}$ the derivations in $\operatorname{Der}(\wedge V, B ; \phi)$ as defined above. Then $\delta \theta_{p}\left(y_{p}\right)=0$ and $\delta \alpha_{q}\left(w_{q}\right)=0$. Since $\iota$ is a homotopy monomorphism, it follows from [7, Theorem 2.2] and [10, Corollary 2.3] that the $G$-sequence of the $\mathrm{CW}$-pair $(Y, X)$ is exact. Thus, $\left[\theta_{p}\right], \ldots,\left[\theta_{k}\right]$ and $\left[\alpha_{q}\right], \ldots,\left[\alpha_{r}\right]$ are the only non-zero homology classes in $H_{*}(\operatorname{Der}(\wedge V, B ; \phi))$. Also, $H\left(\varepsilon_{*}\right)\left(\left[\theta_{p}\right]\right)=\left[y_{p}^{*}\right], \ldots, H\left(\varepsilon_{*}\right)\left(\left[\theta_{k}\right]\right)=\left[y_{k}^{*}\right.$ where $y_{p}^{*} \in \operatorname{Der}(\wedge V, \mathbb{Q} ; \varepsilon)$ and $H\left(\varepsilon_{*}\right)\left(\left[\alpha_{q}\right]\right)=\left[w_{q}^{*}\right], \ldots, H\left(\varepsilon_{*}\right)\left(\left[\alpha_{r}\right]\right)=\left[w_{r}^{*}\right]$ where $w_{q}^{*} \in \operatorname{Der}(\wedge V, \mathbb{Q} ; \varepsilon)$. Hence, $G_{*}(\wedge V, B ; \phi)$ is generated by $\left\langle\left[y_{p}^{*}\right], \ldots,\left[y_{k}^{*}\right]\right\rangle$ and $\left\langle\left[w_{q}^{*}\right], \ldots,\left[w_{r}^{*}\right]\right\rangle$ as a vector space.

Theorem 2.8 Consider the homotopy monomorphism $\iota: X \hookrightarrow Y$ between simply connected formal homogeneous spaces for which $\pi_{*}(Y) \otimes \mathbb{Q}$ is finite dimensional, and let $\phi: \wedge V=\left(\wedge\left(V_{0} \oplus V_{1}\right), d\right) \rightarrow(B, d)$ be its Sullivan model. Then

(i) $G_{*}^{r e l}(\wedge V, B ; \phi)$ is generated as a vector space by $\left\langle\left[\left(y_{k}^{*}, 0\right)\right],\left[\left(0, y_{p}^{*}\right)\right]\right\rangle$ and $\left\langle\left[\left(0, y_{p}^{*}\right)\right]\right\rangle$ for $p \in\{1, \ldots, k\}$ which is determined by the homotopy monomorphism.

(ii) $G_{*}^{r e l}(\wedge V, B ; \phi)$ is generated as a vector space by $\left\langle\left[\left(y_{k}^{*}, 0\right)\right],\left[\left(0, w_{q}^{*}\right)\right]\right\rangle$ and $\left\langle\left[\left(0, w_{q}^{*}\right)\right]\right\rangle$ for $q \in\{1, \ldots, r\}$ which is determined by the homotopy monomorphism. 
Proof. (i) Let $\phi: \wedge V=\left(\wedge\left(V_{0} \oplus V_{1}\right), d\right) \rightarrow(B, d)$ be a Sullivan model for the homotopy monomorphism $\iota: X \hookrightarrow Y$ where $V$ is finite dimensional. Consider a minimal Sullivan model $(B, d)=\left(\wedge\left(V_{0} \oplus V_{1}\right), d\right)$ of $X$ where $\pi_{*}(X) \otimes \mathbb{Q}$ is finite dimensional. Suppose that $V_{1}=V_{1}^{\text {odd }}=\mathbb{Q}<y_{1}, \ldots, y_{k}>$ and define the derivations $\alpha_{t}=\left(y_{t}, 1\right)$ for $t \in\{1, \ldots, k\}$ in $\operatorname{Der}(B, B ; 1)$ and $\theta_{p}=\left(y_{p}, 1\right)$ for $p \in\{1, \ldots, k\}$ in $\operatorname{Der}(\wedge V, B ; \phi)$. Here, the subscripts indicate degrees. Since $\iota$ is a homotopy monomorphism, and the $G$-sequence of the CW-pair $(Y, X)$ is exact [7, 10], we have

$$
\phi^{*}\left(\alpha_{t}\right)= \begin{cases}\theta_{p}, & t=p \\ 0, & t \neq p\end{cases}
$$

such that

$$
D\left(\alpha_{t}, 0\right)= \begin{cases}\left(0, \theta_{p}\right), & t=p, \\ (0,0), & t \neq p,\end{cases}
$$

and $D\left(0, \theta_{p}\right)=(0,0)$. Thus, for $t \neq p,\left[\left(\alpha_{t}, 0\right)\right]$ and $\left[\left(0, \theta_{p}\right)\right]$ are the non-zero homology classes in $H_{*}\left(\operatorname{Rel}\left(\phi^{*}\right)\right)$. Moreover, $H\left(\varepsilon_{*}, \varepsilon_{*}\right)\left(\left[\left(\alpha_{t}, 0\right)\right]\right)=\left[\left(y_{t}^{*}, 0\right)\right]$ for $t \neq p$, and $H\left(\varepsilon_{*}, \varepsilon_{*}\right)\left(\left[\left(0, \theta_{p}\right)\right]\right)=\left[\left(0, y_{p}^{*}\right)\right]$. It is easy to check that for $t \neq p$, $\left[\left(y_{t}^{*}, 0\right)\right]$ and $\left[\left(0, y_{p}^{*}\right)\right] \operatorname{span} H\left(\varepsilon_{*}, \varepsilon_{*}\right)$. On the other hand, we observe that, if $p \neq 1$ for $k=1$, then one recovers non-zero homology classes $\left[\left(\alpha_{t}, 0\right)\right], t \neq p$ and $\left[\left(0, \theta_{p}\right)\right]$ in $H_{*}\left(\operatorname{Rel}\left(\phi^{*}\right)\right)$. However, if $p=1$ for $k=1$, then $\phi^{*}\left(\alpha_{t}\right)=\theta_{p}$ for $t=p$. Thus, $D\left(\alpha_{t}, 0\right)=\left(0, \theta_{p}\right)$ and $D\left(0, \theta_{p}\right)=(0,0)$. It follows that $H\left(\varepsilon_{*}, \varepsilon_{*}\right)\left(\left[\left(0, \theta_{p}\right)\right]\right)=\left[\left(0, y_{p}^{*}\right)\right]$ and $\left[\left(0, y_{p}^{*}\right)\right] \operatorname{span} H\left(\varepsilon_{*}, \varepsilon_{*}\right)$.

(ii) The proof for this part is similar to the one of $(i)$.

From the theorem above, it follows that the $G$-sequence reduces to

$$
\begin{gathered}
0 \rightarrow G_{p}(\wedge V, B ; \phi) \stackrel{H(J)}{\stackrel{H}{\longrightarrow}} G_{p}^{r e l}(\wedge V, B ; \phi) \rightarrow 0, \\
0 \rightarrow G_{k=p}(B) \stackrel{H\left(\phi^{*}\right)}{\longrightarrow} G_{p}(\wedge V, B ; \phi) \rightarrow 0, \\
0 \rightarrow G_{k}^{r e l}(\wedge V, B ; \phi) \stackrel{H(P)}{\stackrel{(\longrightarrow}{\longrightarrow}} G_{k}(B) \rightarrow 0,
\end{gathered}
$$

and it is exact.

\section{Acknowledgement}

This work was completed with the support of the Botswana International University of Science and Tecnnology (BIUST). 


\section{References}

[1] Y. Félix, S. Halperin, and J.-C. Thomas, Rational homotopy theory, Springer, New York, (2001).

[2] Y. Félix, J. Oprea, and D. Tanré, Algebraic models in geometry, Oxford University Press, New York, (2008).

[3] D.H. Gottlieb, Evaluation subgroups of homotopy groups. Am. J. Math., 91 (1969), pp. 729-756.

[4] G. Lupton and S.B. Smith, Rationalized evaluation subgroups of a map. I. Sullivan models, derivations and $G$-sequences. J. Pure Appl. Algebra., 209 (2007), no. 1, pp. 159-171. https://doi.org/10.1016/j.jpaa.2006.05.018

[5] O. Maphane, Derivations of a Sullivan model and the rationalized $G$ sequence. Int. J. Math. Math. Sci., (2021), Art. ID 6687527, 5 pp.

[6] P.A. Otieno, J.-B. Gatsinzi, and O.V. Otieno, Rationalized evaluation subgroups of mapping spaces between complex Grassmannians. Afr. Mat., 31 (2020), pp. 297-303. https://doi.org/10.1007/s13370-01900724-w

[7] J.Z. Pan and M.H. Woo, Exactness of $G$-sequences and monomorphisms. Topology Appl., 109 (2001), pp. 315-320.

[8] D. Sullivan, Infinitesimal computations in topology. Publ. Math. IHES., 47 (1977), pp. 269-331.

[9] E.H. Spanier, Algebraic topology, Springer-Verlag, New York, (1989). Corrected reprint of the 1966 original.

[10] M.H. Woo and K.Y. Lee, On the relative evaluation subgroups of a CW-pair. J. Korean Math. Soc., 25 (1988), no. 1, pp. 149-160.

Oteng Maphane

Department of mathematics and statistical sciences,

Botswana International University of Science and Technology

Private Bag 16, Palapye, Botswana.

maphaneot@biust.ac.bw

Please, cite to this paper as published in

Armen. J. Math., V. 14, N. 2(2022), pp. 110

https://doi.org/10.52737/18291163-2022.14.2-1-10 